\title{
The Modification of Difference Vegetation Index (DVI) in middle and late growing period of winter wheat and its application in soil moisture inversion
}

\author{
Zhang Hong-wei ${ }^{1,2 *}$, Chen Huai-liang ${ }^{2}$, Zha Fei-na ${ }^{2}$ \\ ${ }^{1}$ CMA . Henan Key Laboratory of Agro-meteorological Safeguard and Applied Technique, Zhengzhou 450003; \\ ${ }^{2}$ Henan Meteorological Service, Zhengzhou 450003
}

\begin{abstract}
In the middle and late growing period of winter wheat, soil moisture is easily affected by saturation when using MODIS data to retrieve soil moisture. In this paper, in order to reduce the effect of the saturation caused by increasing vegetation coverage in middle and late stage of winter wheat, the Difference Vegetation Index (DVI) model was modified with different coefficients in different growth stages of winter wheat based on MODIS spectral data and LAI characteristics of variation. LAI was divided into three stages, $\mathrm{LAI} \leq 1<\mathrm{LAI} \leq, 3<\mathrm{LAI}$, and the adjusting coefficient of $\mathrm{a}=1, \mathrm{a}=3, \mathrm{a}=5$, were taken to modifying the Difference Vegetation Index(DVI). The results show that the Modified Difference Vegetation Index (MDVIa) can effectively reduce the interference of saturation, and the inversion result of soil moisture in the middle and late period of winter wheat growth is obviously superior to the uncorrected inversion model of DVI.
\end{abstract}

\section{Introduction}

Remote sensing monitoring of soil moisture and drought is a frontier field of remote sensing technology and application research, and it is recognized as one of the world research problems. the exploration and research in soil moisture data and its application were relative active for its high application value to agriculture, hydrological, meteorology and so on. Drought is the most important natural disaster in agriculture in China, and has become the most serious meteorological disaster affecting agricultural production in China. Drought has the highest frequency, the longest duration, the largest range of influence, and the heaviest direct loss to agricultural production. In comparation with other natural disasters. The study of objective, dynamic and real-time monitoring methods of soil moisture and drought is helpful in taking positive and effective prevention and anti-measures. There are two main methods of soil moisture and drought monitoring, one is to use the network of ground observation stations to monitor soil moisture. The common methods are: Weighing drying method, Neutron method, TDR method and so on. The main advantages of these monitoring methods are that the single point measurement accuracy is high, the shortage is the limited sampling point and the soil characteristics are not uniform, the single point data is difficult to represent the large area condition, at the same time, the manpower and material resources are also large. Another soil moisture monitoring method is satellite remote sensing inversion method, which has the characteristics of wide coverage and strong real-time.
Drought is caused by the long-term deficit of soil water content, and as a natural disaster, the drought will have a great impact on agriculture, ecology and social economy, especially on agricultural production. An aggravating trend of winter wheat drought in North China after 1990s was founded by Wu Dong-li et al. whose study focus on the temporal and spatial distribution characteristics of winter wheat drought in North China. Therefore, drought monitoring, forecasting, early warning, evaluation and so on have aroused serious concern of scientific research departments and government decisionmaking.

In the field of remote sensing inversion of soil moisture, many soil moisture inversion models were proposed based on vegetation index (VI), and these vegetation index (VI) were constructed mainly adopts the reflectivity of Near Infrared and Infrared bands. So, these soil moisture inversion models are intuitionistic and easy to use, and can reflect the change of soil moisture in a certain depth through vegetation index. These indexes can reflect the water content information of vegetation effectively, for they are changing when the water stress was put on the vegetation. This is of great theoretical and practical significance for the monitoring of drought change and development.

The amount of soil water content changes the vegetation coverage and the reflectivity of the underlying surface, thus affecting the energy cycle of vegetation, soil and atmosphere. The pre-study results showed that the lower the soil moisture is, the smaller the vegetation index is and the higher the spectral reflectivity is. Therefore, it is possible to find a better characteristic index to reflect

* Corresponding author: xxqxjzhw1966@163.com 
the temporal and spatial variation of soil moisture by synthesizing these factors. At the same time, through extensive application, it is found that the soil moisture inversion model based on vegetation index (VI) is vulnerable to saturation with the increase of vegetation coverage. For example, Jiang-dong et al studied drought in the main winter wheat producing areas in northern China by using anomaly vegetation index (AVI) and anomaly water index (AWI). It was found that the response of anomaly water index (AWI) to drought was more sensitive and accurate than that of anomaly vegetation index (AVI). In this way, the vegetation index cannot be directly used to reflect the real change of soil moisture, which seriously affects the inversion accuracy of soil moisture.

The study results showed that the estimation of the continuous variation of vegetation canopy by using the satellite sequence data of MODIS mainly depends on the empirical statistical correlation of the single spectral vegetation index based on red light and near infrared spectrum inversion. It is found that the high reflectivity and transmittance of vegetation crown leaves, especially wide leaves, in the near infrared spectrum region can cause strong multiple scattering. With the increase of leaf area index, multiple scattering will increase rapidly. In this case, the sensitivity of reflectivity in one or more directions to different parameters is weakened. Therefore, spectral vegetation index is easy to reach saturation when the leaf area index of vegetation is lower than 2-3. This saturation effect limits the effective inversion imaging of ground physical parameters to vegetation coverage, so now researchers make full use of different spectral information to construct different vegetation index to reverse vegetation leaf area index. However, most of these models contain many parameters, and a large number of parameters and rich meteorological and agricultural background knowledge are needed to run the models. Therefore, these models are not suitable for regional large-scale and real-time inversion.

In this paper, the Difference Vegetation Index (DVI)of winter wheat in different growth stages was modified which according to the characteristics of leaf area index (LAI)change in Henan Province, combined with satellite remote sensing monitoring data, based on the infrared and near-infrared spectroscopy of MODIS. And the soil moisture was inversely with different models, the results show that the Difference Vegetation Index (DVI)can improve the soil moisture inversion accuracy effectively in the middle and later stage of winter wheat, and can better reflect the variation of soil moisture.

\section{Data \& Methods}

\subsection{Study Area \& Data}

In this paper, Henan Province is selected as the study area, the geographical span is $31^{\circ} 23 \leqslant 36^{\circ} 22^{\circ} \mathrm{N}, 110^{\circ} 22 \leqslant$ $116^{\circ} 39 \mathrm{E}$. the soil moisture in the period of return to maturity of winter wheat is selected be the research object. The soil moisture data come from the daily mean value of
$0-50 \mathrm{~cm}$ soil moisture in Henan automatic soil moisture observation Station.

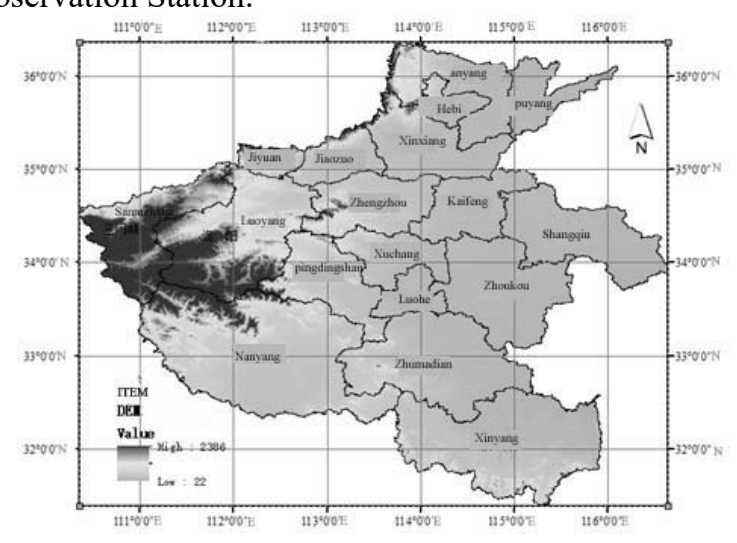

Fig1. Map of Henan Province

The remote sensing data, which format is LD3, were collected from March to May 2018 from satellite station of Henan Institute of Meteorological Sciences. The spectral data of the field was observed at the fixed point of ENVI4.7 from the first ten days of Mar. to the last ten days of May, and the Difference Vegetation Index (DVI) was calculated and reversed in soil moisture by using the infrared light (610-680 nm, the first wave band corresponding to the MODIS), the near-infrared light (NIR) (780-890 nm, the second wavelength band corresponding to the MODIS).

\subsection{Methods}

MODIS is a new generation of optical remote sensing instruments in the world, which has 36 spectral channels. The characteristics of EOS/MODIS sensor, such as high time resolution, high spectral resolution and moderate spatial resolution, make it have outstanding advantages in the dynamic change monitoring of crop growth. The study results showed that there is a low reflectance region at 675 $\mathrm{nm}, 1450 \mathrm{~nm}$ and $1900 \mathrm{~nm}$ bands, i.e. absorption peak region, and a high reflectance region at $855 \mathrm{~nm}, 1650 \mathrm{~nm}$ bands. At the same time, according to the spectral reflectance curve of winter wheat measured in China, under different leaf area indexes, the greater the leaf area index is, the greater the absorption rate is, the smaller the reflectance is. It can be seen that the different leaf area index (LAI) is an important factor to influence the change of the spectral curve, and this also provides a possibility for the correction of the soil moisture inversion model using the MODIS spectral data by using the change of the leaf area index. 


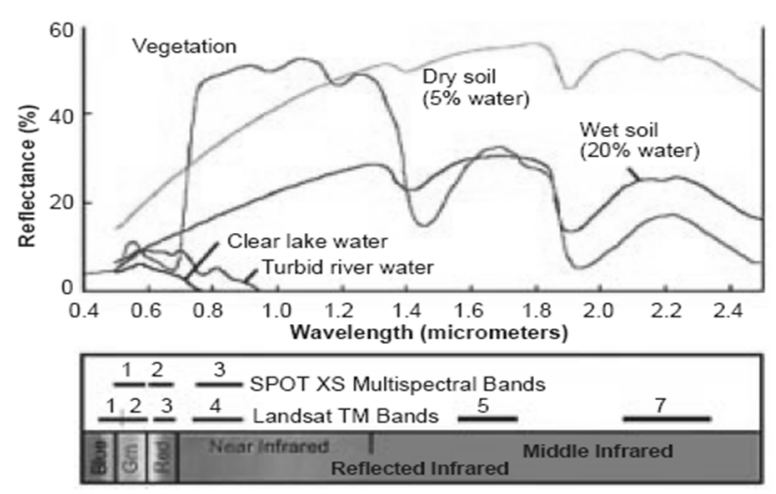

Fig.2 Spectral Reflectance curves for vegetation, soil and water

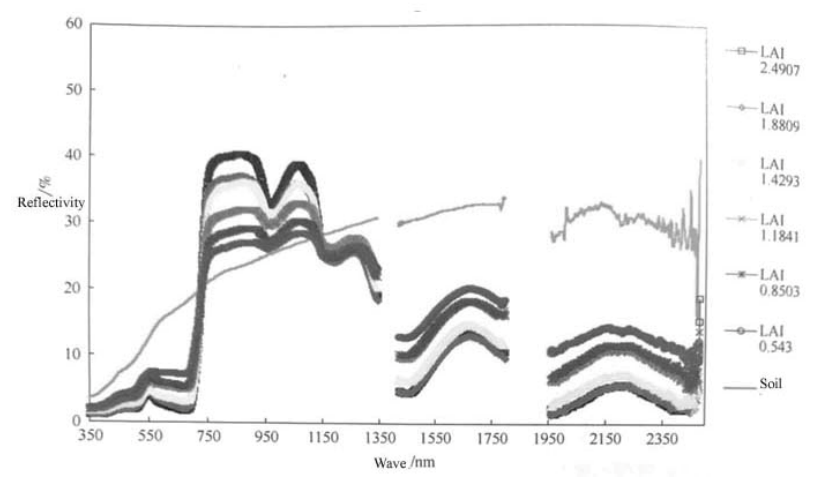

Fig. 3 Spectral Reflectance curves in Varies LAI

\subsection{1 the change of leaf area index in different growth stages of winter wheat}

The leaf area index (LAI) of winter wheat is a continuous process with the growth period, but there are obvious stage features and turning points. In the early stage of growth (emergence-overwintering), the leaf area index (LAI) increased slowly, basically in linear growth; during the overwintering, the winter wheat basically stopped growing. After returning to the green, the winter wheat entered the stage of vegetative growth and reproductive growth, and the leaf area index (LAI) increased rapidly and was in the form of a classical Logistic curve until the winter wheat was mature. According to the variation characteristics of leaf area index during wheat growth, the change characteristics of leaf area index after turning green of winter wheat are shown in Fig. 4. The change equation is as follows:

$$
L A I=\frac{a}{b+E X P\left(c+d * t+e * t^{2}\right)}
$$

There, LAI is the leaf area index, $t$ is the number of days after the return of winter wheat, and a, b, c, d and e are coefficient.

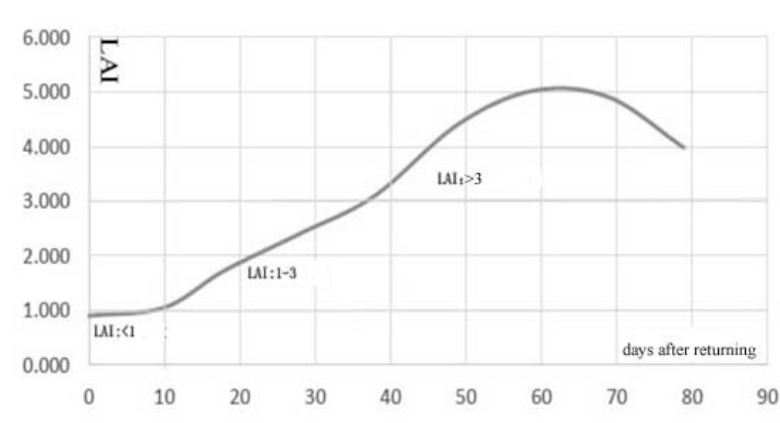

Fig.4 The LAI curve of Winter Wheat from the stage of Return Green to Ripe

\subsubsection{Method for correcting difference vegetation index (DVI) based on MODIS spectral data}

It is proved that the green vegetation has strong absorption characteristics in the red-light region. Moreover, the larger the population is, the lower the absorption rate is in the red light region, but the decrease was only $3 \% \sim 5 \%$, and On the contrary, it could reach $40 \% \sim 60 \%$ in the infrared region. Li Xin-chuan et al. (2012) analyzed the sensitivity of vegetation index to leaf area index under different soil parameters and showed, the reflectivity of red light and near infrared band changed obviously when LAI $\leqslant 3$, the reflectivity change of redlight band was basically the same, while the near infrared band was obviously affected by soil change when LAI value $>3$, the reflectivity change of near infrared band decreased gradually when LAI $>5$. Therefore, the winter wheat growth period is divided into three stages, namely, $\mathrm{LAI} \leq 1,1<\mathrm{LAI} \leq 3$ and $\mathrm{LAI}>3$ stage, in combination with the change of the leaf area index during the return-tomaturity period of the winter wheat given in 2.2.1.
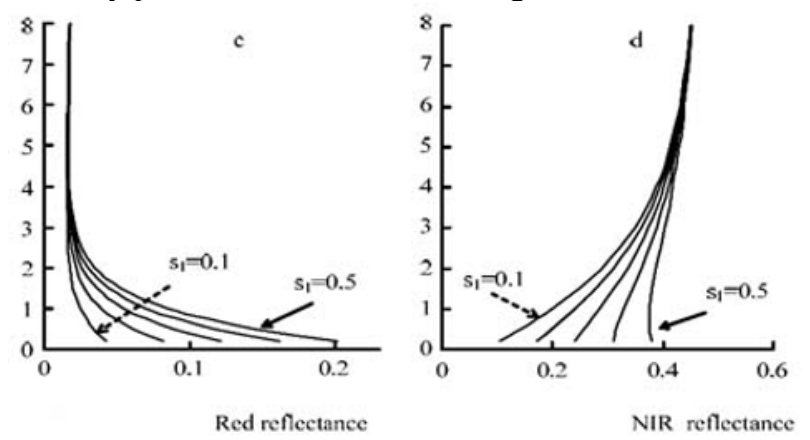

Fig5. Correlation between LAI and Red, Nir and VI under different soil parameters

In this paper, the difference vegetation index (DVI) is used to modify the vegetation index. The model is as follow:

$$
D V I=B_{2}-B_{1}
$$

There, $\mathrm{B} 1$ and $\mathrm{B} 2$ correspond to the reflectivity of each band of MODIS channel 1 and channel 2, respectively.

According to the change of leaf area index of winter wheat described in 1.2.1, the first stage of Henan province was defined as the winter wheat jointing stage before (before March 10, after the return of winter wheat to about 10 days), and the leaf area index (LAI) $<1$; The second 
stage is the stage of winter wheat heading before (from March 11 to April 10, between 11 and 40 days after the return of winter wheat), and the leaf area index is mainly in the stage of 1-3; and the third stage is the headingflowering of winter wheat (from April 11 to May 10). After 41 days after the return of winter wheat, the leaf area index was at the stage of $>3$ (FIG.4). The difference vegetation index (DVI) was revised according to the three stages of the leaf area index of winter wheat, as follows:

$$
M D V I_{\mathrm{a}}=\mathrm{a} * B_{2}-B_{1}
$$

There, the correction factor a for different developmental stages of winter wheat is set as 1, 3 and 5 respectively according to the three stages.

\section{Results \& Validation}

\subsection{Terrain and landform and drought occurrence in Henan Province}

Henan is located in the middle and east of China and the middle and lower reaches of the Yellow River. It covers an area of 167000 square kilometres, the boundary is $31^{\circ} 23^{\prime} \sim 36^{\circ} 22^{\prime} \mathrm{N}$ and $110^{\circ} 21^{\prime} \sim 116^{\circ} 39^{\prime} \mathrm{E}$. The terrain and topography of the east and west are very different. For it is located in the warm temperate zone and the northern subtropical transitional zone, a typical continental monsoon climate has been formed. The rain and heat are in the same season, and the climate is significantly different from the north to the south.

The frequency of spring drought and early summer drought in Henan Province accounts for more than 55\% of all drought, which is about once in 3 years. This period coincides with the peak period of vegetative growth of winter wheat in Henan Province, and the amount of precipitation in this period will directly affect the yield of wheat. Especially in recent years, with the acceleration of global warming, extreme climate events occur frequently. The precipitation distribution in Henan province from March to May showed significant variability, and the precipitation in most of the regions showed a tendency to decrease, and the reduction rate was between $3 \% \sim 20 \%$. Therefore, it was more necessary to study the drought monitoring during the middle and later stages of the growth of wheat

\subsection{Validation}

Different drought indexes can provide different monitoring information at different time and ecosystem, and remote sensing monitoring information should contain different underlying surface conditions. In order to compare the effectiveness of soil moisture inversion after the modification of difference vegetation index (DVI) in middle and late stage of winter wheat, the satellite remote sensing monitoring data of five different phases, March 25, 2018, April 1, 2018, April 19, 2018, May 7, 2018 and May 12, 2018, were selected for study in the model of no modified(DVI) and modified of differential vegetation index (MDVI). At the same time, the soil weight water content of $0 \sim 50 \mathrm{~cm}$ from soil moisture observation station of meteorological station in the whole province was used to verified the effectiveness of modified difference vegetation index (MDVI).

The difference vegetation index (DVI) of five remote sensing images were extracted and the formula (3) was used to modify the difference vegetation index (DVI), and the MDVI was obtained, and the correlation analysis and significance test were carried out with the daily mean value of soil weight water content of depth $0-50 \mathrm{~cm}$ observed from an automatic soil water sub-station. It can be seen that at different stages, the modified difference vegetation index (MDVI $\alpha$ ) has higher correlation with the daily average soil weight water content at depth of $0 \sim 50 \mathrm{~cm}$, and all pass the significance test with $\alpha=0.01$. Therefore, the modified difference vegetation index (MDVI) method has higher accuracy when monitoring the soil moisture at the level of $0-50 \mathrm{~cm}$ in the middle and late stage of the growing of the crops with large vegetation coverage.

The distribution maps of DVI, MDVI and soil weight water content data of five phases in ArcGIS were made by Kriging interpolation method (Fig. 6). It can be seen that the modified difference vegetation index (MDVI) can better reflect the changes of soil water under high cover in the middle and late growth period of winter wheat.

Table1 Regression and significance Test of Difference Vegetation Index(DVI) before and after modification

\begin{tabular}{|c|c|c|c|c|c|c|}
\hline Date & Index & Regression equation & Sample No. & $\mathbf{R}^{2}$ & $\mathbf{F}$ & Prob $>F$ \\
\hline \multirow{2}{*}{ March 25, 2018} & DVI & $y=0.0253 x+15.744$ & 80 & 0.1982 & 20.3 & 0.05 \\
\hline & $\mathrm{MDVI}_{\mathrm{a}}$ & $y=0.0144 x+7.2597$ & 80 & 0.5108 & 85.6 & 0.01 \\
\hline \multirow{2}{*}{ April 1, 2018} & DVI & $y=0.0149 x+15.413$ & 80 & 0.0799 & 6.8 & 0.05 \\
\hline & $\mathrm{MDVI}_{\mathrm{a}}$ & $y=0.0097 x+9.0319$ & 80 & 0.5131 & 83.1 & 0.01 \\
\hline \multirow{2}{*}{ April 19, 2018} & DVI & $y=0.0227 x+13.097$ & 80 & 0.4927 & 62.9 & 0.01 \\
\hline & $\mathrm{MDVI}_{\mathrm{a}}$ & $y=0.006 x+8.753$ & 80 & 0.4904 & 70.2 & 0.01 \\
\hline \multirow{2}{*}{ May 7, 2018} & DVI & $y=0.0032 x+16.377$ & 80 & 0.0020 & 0.1 & 0.05 \\
\hline & $\mathrm{MDVI}_{\mathrm{a}}$ & $y=0.007 x+3.9905$ & 80 & 0.4966 & 68.1 & 0.01 \\
\hline \multirow{2}{*}{ May 12, 2018} & DVI & $y=0.0015 x+14.779$ & 80 & 0.0050 & 0.1 & 0.05 \\
\hline & $\mathrm{MDVI}_{\mathrm{a}}$ & $y=0.0078 x+1.7787$ & 80 & 0.4926 & 64.1 & 0.01 \\
\hline
\end{tabular}



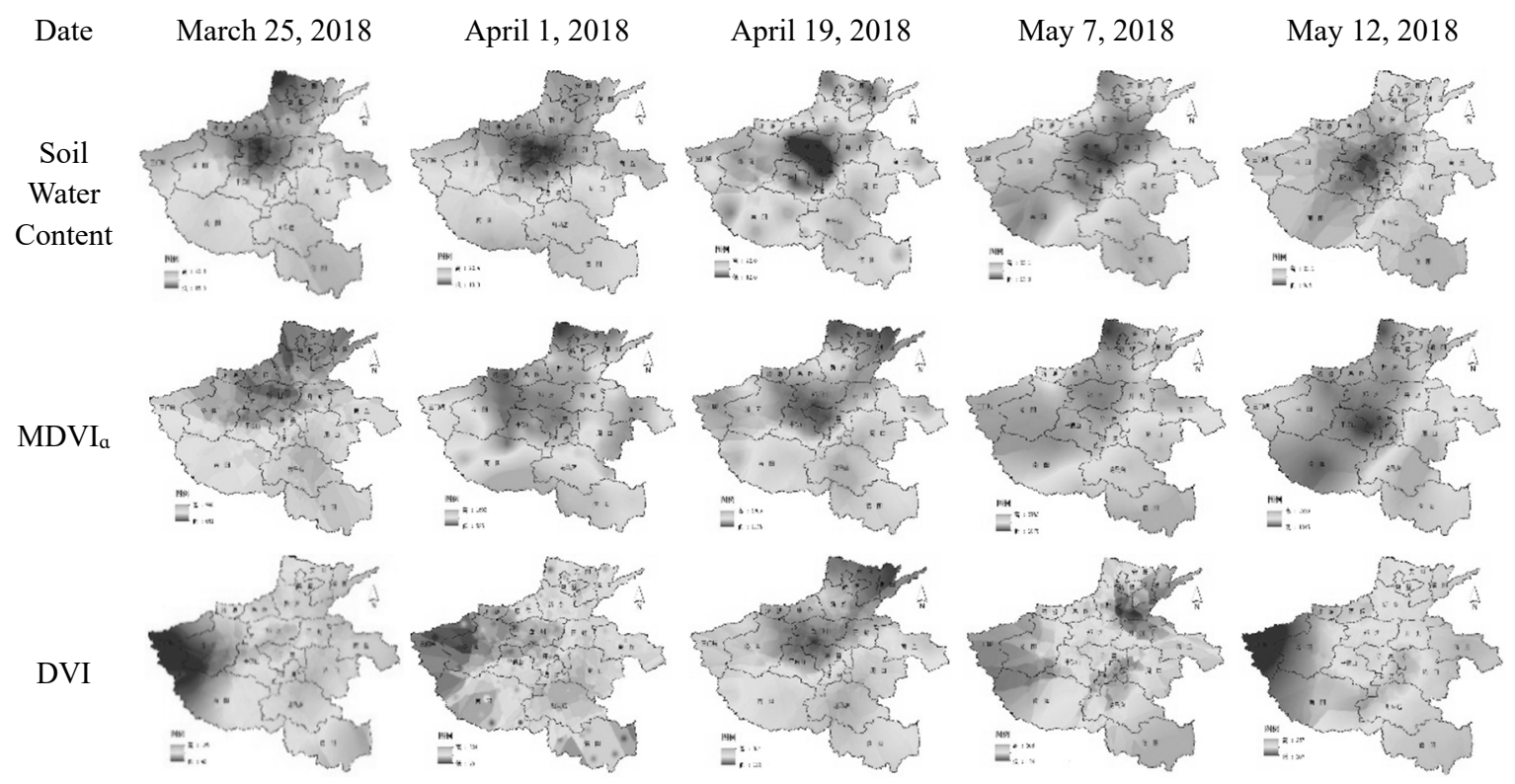

Fig. 6 The Sketch Map of Soil Moisture, MDVIa、DVI in 5 Time Phases

\section{CONCLUSIONS \& DISCUSSIONS}

(1) The modified difference vegetation index can effectively reduce the disturbance of saturation caused by the increase of vegetation coverage on the remote sensing inversion accuracy of soil moisture, and the inversion result of soil moisture in the middle and late growth period of winter wheat is obviously better than that of unmodified inversion accuracy.

(2) The soil moisture inversion in the middle and late stage of winter wheat growth was feasible with LAI was divided into three stages, $\mathrm{LAI} \leq 1<\mathrm{LAI} \leq, 3<\mathrm{LAI}$, and the adjusting coefficient of $a=1, a=3, a=5$, were taken to modifying the Difference Vegetation Index (DVI).

The geographical span of Henan Province is large, the climate condition is different from the north and the south, the topography of the east and west and the soil texture are different, and the regional division is necessary in the future research, which is beneficial to the further improvement of the precision of the drought inversion. At the same time, only the difference vegetation index (DVI) is studied, and the application of other indexes is to be studied deeply.

\section{References}

1. Huang Zhong-xiong, Su Yong-xiu, Zhou Jian-bo, et al. Study on the soil moisture measurement techniques[J]. Journal of Meteorological Research and Application, 2014, 35(4):58-62.

2. WU Dongli1, WANG Chunyi, XUE Hongxi, et al. Temporal and spatial distribution of drought for winter wheat in north China[J]. JOURNAL OF NATURAL DISASTERS, 2012,21(1):18-25.

3. Nemani, R., Price, L., Running, S., Goward, S., 1993. Developing satellite derived estimates of surface moisture status. Journal of Applied Meteorology 32 (3), 548-557.

4. Jiang Dong, Yuan Fu-jing, Zhuang Da-fang, et al. Dynamic Drought remote sensing monitoring in north China from 2008 to 2009[J]. JOURNAL OF NATURAL DISASTERS, 2012, 21(3):92-101.

5. Di, L., Rundquist, D.C., Han, L., 1994.Modeling relationships between NDVI and precipitation during growth cycle. International Journal of Remote Sensing 15 (10), 2121-2136.

6. Kogan, F.N., 1990. Remote sensing of weather impacts on vegetation in non-homogeneous areas. International Journal of Remote Sensing 11 (8), 1405-1419.

7. Liu, W., Ferreira, A., 1991. Monitoring crop production regions in the Sao Paulo State of Brazil using normalized difference vegetation index. Proc. 24th. International Symposium on Remote Sensing of Environment, Rio de Janeiro, Brazil, vol. 2, pp. 447-455. 27-31 May.

8. Peters, A.J., Walter-Shea, E.A., Lei, J., Vina, A., Hayes, M., Svoboda, M.R., 2002. Drought monitoring with NDVI-based standardized vegetation index. Photogrammetric Engineering and Remote Sensing 65 (1), 71-75.

9. Unganai, L.S., Kogan, F.N., 1998. Drought monitoring and corn yield estimation in Southern Africa from AVHRR data. Remote Sensing of Environment 63 (3), 219-232.

10. Yang, L., Wylie, B.K., Tieszen, L.L., Reed, B.C., 1998. An analysis of relationships among climate forcing and time-integrated NDVI of grasslands over the U.S. northern and central Great 
Plains. Remote Sensing of Environment 65 (1), 2537.

11. Chen, J.M., Pavlic, G., Brown, L., Cihlar, J., Leblanc, S.G., White, H.P., Hall, R.J., Peddle, D.R., King, D.J., Trofymow, J.A., Swift, E., Van der Sanden, J., Pellikka, P.K.E., 2002. Derivation and validation of Canada-wide coarse-resolution leaf area index maps using high resolution satellite imagery and ground measurement. Remote Sensing of Environment 80 (1), 165-184.

12. M.V.K. Sivakumar, P.S. Roy, K. Harmsen, et al. Satellite Remote Sensing and GIS Applications in Agricultural Meteorology [M]. Published by World Meteorological Organization, 7bis, Avenue de la Paix, 1211 Geneva 2, Switzerland, 2004.

13. Wang Jin-di, Zhang Li-xin, Liu Qin-huo, et al. Typical feature spectrum knowledge base in China[M]. Beijing: Science Press, 2009.

14. Zhang Hong-wei, Chen Huai-liang. The Monitoring Method and Its Application of the Dynamic Change of LAI for Winter Wheat[J]. Journal of Irrigation and Drainage, 2014,33(2):28-31.

15. LIU Zhan-dong DUAN Ai-wang GAO Yang, et al. Study on Dynamic Model of Leaf Area Index(LAI) for Winter Wheat in Xinxiang Area[J], JOURNAL OF TRITICEAE CROPS, 2008,28(4): 680-685.

16. LI Xin-chuan, XU Xin-gang, BAO Yan-song, et al. Retrieving LAI of Winter Wheat Based on Sensitive Vegetation Index by the Segmentation Method[J]. Scientia Agricultura Sinica, 2012,45(17):3486-3496.

17. Liu Liang-ming. The Study of Remote Sensing Models and its application in Drought Pre-Warning based on EOS MODIS data[D]. Wuhang: Wuhang University, 2004.

18. Cheng Bing-yan, Qian Xiao-yan, Zhu Ye-yu. The Process and Frequency Spatial-temporal Distribution of drought in Henan Province in recent 50 years[J]. Henan Meteorology, 1999, (1):24-25.

19. ZHANG Hong-wei, CHEN Huai-liang, ZHANG Hong. Spatiotemporal distribution of precipitation and temperature change in Henan Province in spring: an analysis based on GIS[J]. JOURNAL OF NATURAL DISASTERS, 2012,21(1):191-194. 